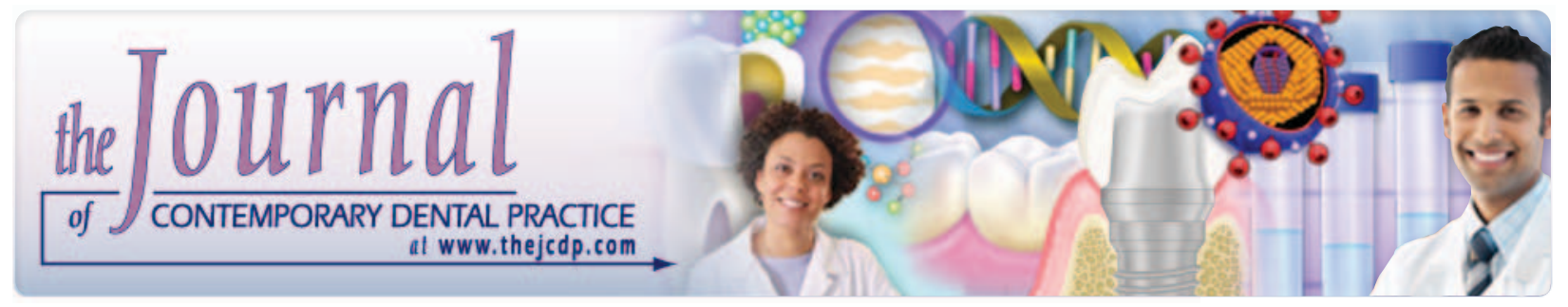

\title{
Porcelain Fused to Metal Crown as an Abutment of a Metal-Ceramic Resin-Bonded Fixed Partial Denture: A Clinical Report
}

Marjaneh Ghavamnasiri, DDS, MS; Fatemeh Maleknejad, DDS, MS; Maryam Modabber, DDS

\begin{abstract}
Aim: This clinical report describes a conventional resin-based fixed partial denture for a 42-yearold woman to close an edentulous space in the region of the second maxillary premolar. While the first maxillary molar had been crowned with porcelain fused to metal (PFM) system 10 years ago and the first premolar was intact and sound except for a small carious lesion in the distal aspect. The essential clinical and laboratory procedures are illustrated.
\end{abstract}

Background: Multiple clinical studies document excellent long-term success of resin-bonded fixed partial dentures. In the 1970s, the Rochette resin-bonded fixed partial denture (RBFPD) was introduced. Next, to enhance retention and resistance form of posterior RBFPD preparation of parallel guide surfaces on the interproximal aspects of the adjacent teeth along with rests on the occlusal aspects to counteract dislodging forces was recommended.

Report: A 42-year-old woman presented with an extracted left maxillary second premolar. The first maxillary molar had been crowned 10 years ago and the first premolar had a small carious lesion in the mid distal of proximal aspect. To eliminate unnecessary cutting of the PFM of the first molar and crown preparation of the first maxillary premolar, the use of RBFPD was suggested and accepted by the patient.

Summary: This clinical report described the indication, the selection of a PFM-crowned

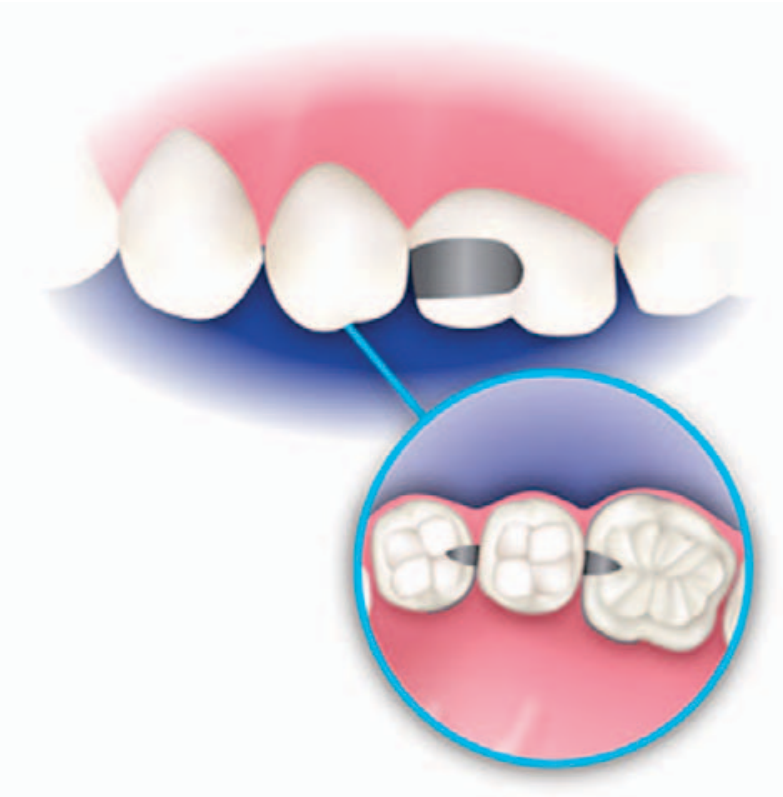

abutment, and clinical procedures involved in the fabrication of RBFPD that provided a conservative solution for replacement of one posterior tooth.

Clinical Significance: RBFPDs can be used successfully in both the anterior and the posterior regions of the mouth to replace one or two missing teeth. However, the survival rate of RBFPDs is still considerably less than that of conventional fixed partial dentures. The principal reason for failure is debonding of the framework from the abutment tooth. The selection of nonmobile abutment teeth, the preparation design that enhances retention and resistance form, and the tooth bonding technique are critical for success. 
Keywords: Resin-bonded fixed partial denture (RBFPD), porcelain fused to metal crown (PFM), bonding, case report

Citation: Ghavamnasiri M, Maleknejad F, Modabber M. Porcelain Fused to Metal Crown as an Abutment of a Metal-Ceramic ResinBonded Fixed Partial Denture: A Clinical Report. J Contemp Dent Pract [Internet]. 2010 March; 11(2):064-070. Available from: http://www.thejcdp. com/journal/view/volume11-issue2-ghavamnasiri.

\section{Introduction}

Replacement of missing teeth remains a challenge in dentistry today. Each treatment has advantages and disadvantages, making a particular restoration suitable only in certain situations. The treatment options depend on the age of the patient, periodontal health, endodontic status, coronal structure, and occlusal relationship. ${ }^{1}$

The insertion of an osseointegrated dental implant is a new and very beneficial solution, with the advantage that unrestored or minimally restored adjacent teeth do not need to be integrated in the restoration. ${ }^{2}$ The specific considerations that may preclude this approach are the availability of bone volume in the edentulous region, occlusal function, systemic disorders, and socioeconomic status of the patient. ${ }^{3}$

The classical approach of fixed partial denture (FPD) is still an option. Unfortunately, conventional bridges require treatment and preparation of a possibly healthy tooth. Therefore, a porcelain fused-to-metal FPD is not an adequate treatment if preservation of dental hard tissue of the abutment teeth is desirable. To preserve the integrity of abutment teeth as much as possible, a conventional RBFPD could be an alternative treatment. The advantage of RBFPDs is its noninvasive approach to dentin with tissue tolerance because of supra-gingival margins. ${ }^{3}$

RBFPDs can be used in both the anterior and posterior regions of the mouth to replace one or two missing teeth. Even though the survival rate of RBFPDs is still considerably lower than that of conventional fixed partial dentures, careful abutment selection, tooth preparation, alloy selection, and bonding technique are critical for clinical success. ${ }^{4}$
Disadvantages of fiber-reinforced RBFPDs include insufficient long-term color stability, low flexural and tensile strength, polymerization shrinkage, low resistance to attrition, and lack of long-term clinical data..$^{5}$

In recent studies, single retainer designs of RBFPDs were inserted in all regions of the arch with acceptable results. $\frac{6-10}{2}$ Clinical indications for RBFPDs include vital and intact abutment teeth, a short edentulous span such as one or two missing teeth, and minimal dynamic occlusal contacts on the abutment teeth. ${ }^{-3}$

This clinical report described a treatment for a missing maxillary second premolar while one of the abutments has a porcelain fused to metal crown.

\section{Clinical Report}

\section{Diagnosis}

A 42-year-old woman presented with a missing left maxillary second premolar that has been extracted one month ago. The first maxillary molar had been crowned 10 years ago and the first premolar had a small carious lesion $(2 \mathrm{~mm}$ wide $\times 2 \mathrm{~mm}$ long $\times$ $1 \mathrm{~mm}$ deep) in the mid distal of proximal aspect. The lateral occlusion of the patient was canine guided and a posterior disclusion was observed on the working side (Figure 1).

Upon radiographic examination, bone loss was observed in the edentulous space as well as vertical distal bone loss at the first premolar (Figure 2).

\section{Treatment}

The following prosthetic treatment options were considered: (1) an implant-supported single crown, (2) a conventional FPD, (3) (RBFPD).

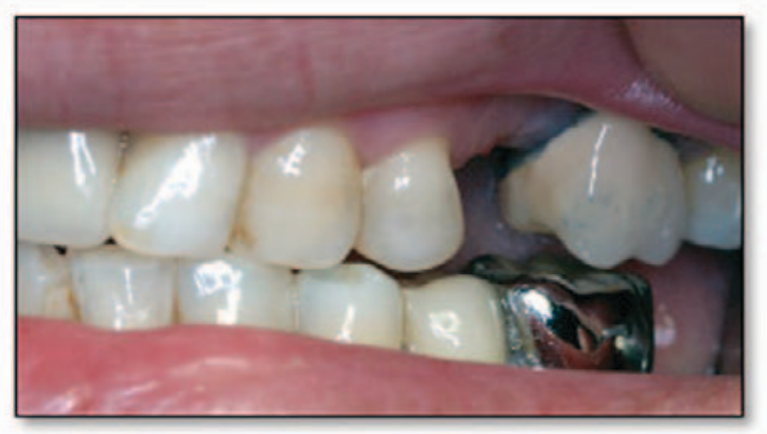

Figure 1. Posterior disclusion on the working side. 


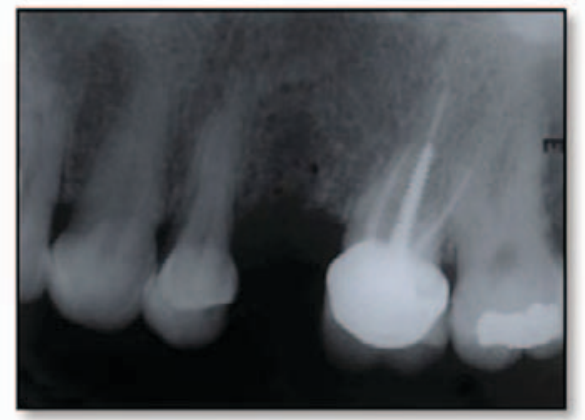

Figure 2. Radiographic view.

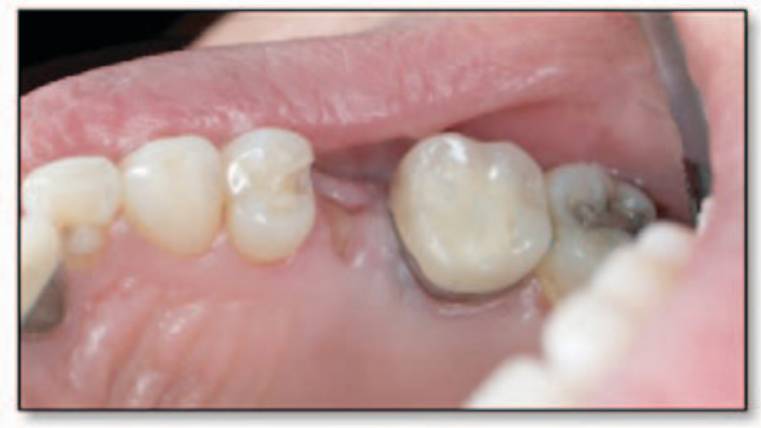

Figure 3. Occlusal rests were prepared in the distal marginal ridge of the first premolar and the mesial marginal ridge of the first molar.

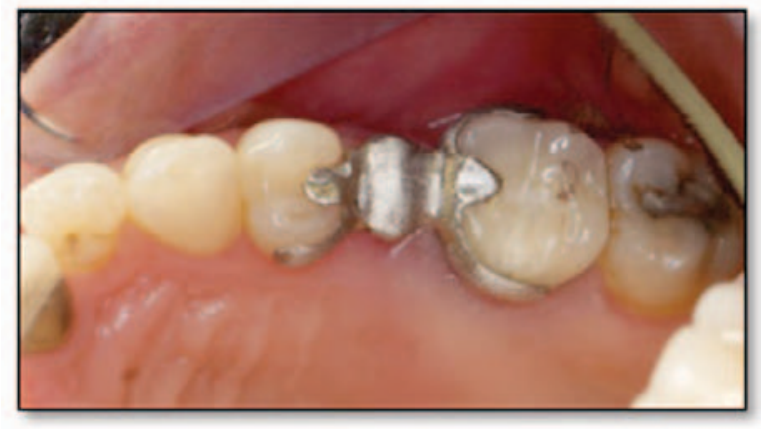

Figure 4. Occlusal view showing the base metal frame of the RBFPD replacing the second premolar.

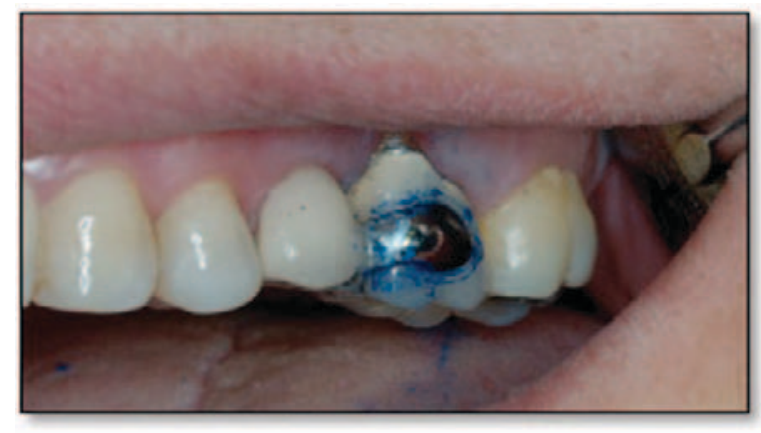

Figure 5. An oil-based marker was used for determination of areas that should be etched with HF.
Implant replacement was excluded as an option because the patient declined the surgical procedure. The conventional bridge also was excluded because the first maxillary molar was crowned and the first premolar was vital. The clinical criteria of a PFM crown including marginal fitness, retention, color match, and contour showed acceptable clinical behavior. Additionally, upon radiographic examination no periapical lesion and periodontal ligament widening were observed (Figure 2). Removing the crown might cause severe damage to the crown foundation and its cutting was a difficult and tiresome procedure. Thus, to eliminate unnecessary cutting of the PFM of the first molar and crown preparation of the first maxillary premolar, the use of RBFPD was suggested and accepted by the patient.

An occlusal rest on the first molar was prepared in the porcelain thickness of the marginal ridge, while for the first premolar it was created in the sound distal marginal ridge tissue. A fine round diamond bur was used for the rest preparation (801012M, NTI-Kahla, Kahla, Germany) (Figure 3).

The distal surface carious lesion of the premolar was prepared with the previously mentioned round diamond bur as a modified class II cavity preparation. 11 After acid etching with 35\% phosphoric acid for 15 seconds, a total-etch onestep adhesive (Excite, Ivoclar/Vivadent, Schaan, Liechtenstein) was applied to the etched enamel and dentin according to the manufacturer's directions and the cavity preparation was filled with a flowable composite (Tetric Flow, Ivoclar/ Vivadent, Schaan, Liechtenstein), $\frac{12,13}{2}$ then light cured with a curing unit (Astralis 7, Ivoclar/ Vivadent, Schaan, Liechtenstein) for 60 seconds. Next, the composite restoration was finished and polished. A condensation silicone material (Speedex, Colten, Switzerland) was applied for the impression procedure, and a cast was formed (DVP, Whip Mix, Louisville, Kentucky, USA). The framework of the RBFPD was fabricated with the lost-wax technique and a base metal alloy (Optimum, Matech, Sylmar, California, USA) (Figure 4). Feldespathic porcelain was used in multiple increments and fired for the construction of the pontic. After trial of fitting and adjusting the occlusal contact areas and glazing of the pontic porcelain, the fitting surface of the retainers was airborne-particle abraded (Hi-Blaster-II, Shofu, Kyoto, Japan) with $50 \mathrm{~m}$ alumina and then conditioned with a silane coupling agent primer 


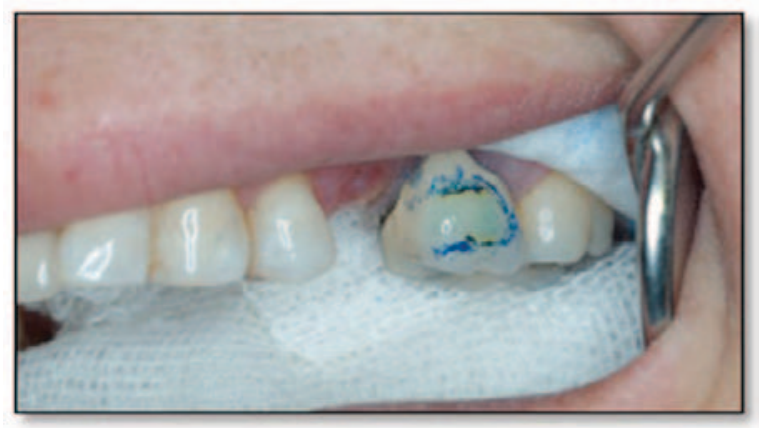

Figure 6. Etching of porcelain crown with HF.

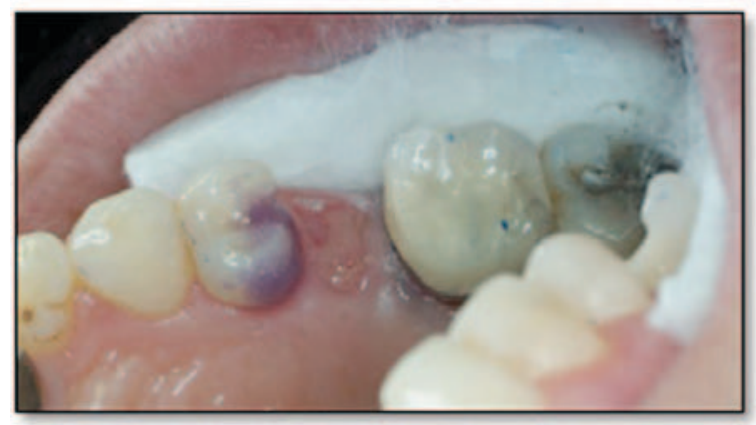

Figure 7. The occlusal rest and lingual aspect of the premolar were etched with phosphoric acid.

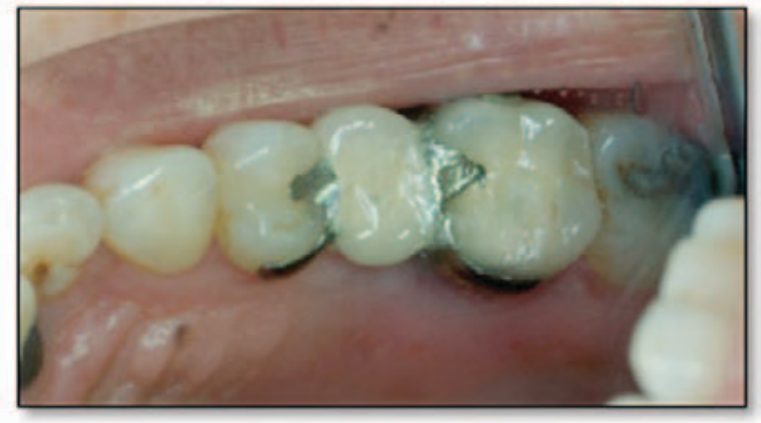

Figure 8. Occlusal view after bonding of RBFPD.

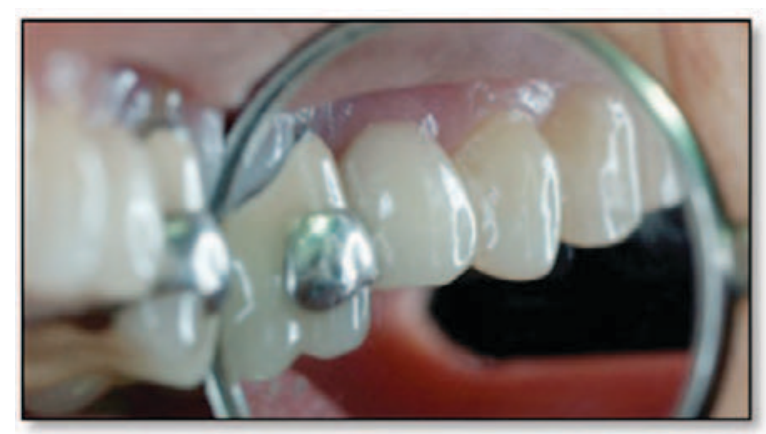

Figure 9. Buccal view of the bridge.
(Ceramic Primer, 3M ESPE, St. Paul, Minnesota, USA) designed for porcelain. The prosthesis was inserted and an oil-based marker outlined around the buccal and lingual retainers of the first maxillary molar to confirm the exact sites of the etching material (Figure 5).

After removing the bridge, the mentioned areas on the buccal and lingual walls of the porcelain crown were etched with a hydrofluoric acid gel (Ultradent Products Inc., South Jordan, Utah, USA) for 2 minutes, while the enamel surfaces of the first premolar were etched with a $35 \%$ phosphoric acid (Ultra-Etch, Ultradent Products Inc., South Jordan, Utah, USA) for 15 seconds (Figures 6 and 7). The application of ED primer was followed by the luting with an adhesive dual-polymerizing resin cement (Panavia F, Kuraray, Osaka, Japan) according to the manufacturer's directions and light cured and then the excess resin cement material was eliminated (Figures 8 and 9).

\section{Discussion}

The advantages of replacing missing teeth with RBFPDs include conservation of tooth structure, lack of pulp irritation, minimal periodontal involvement, reversibility, and reduced cost. ${ }^{14}$

According to the method of Kaplan and Meier, the four-year success rate of the various designs decreased to $74 \% \pm 2 \%$ for 1598 RBFPDs compared with $74 \% \pm 2 \%$ at 15 years for 4118 conventional fixed partial dentures. ${ }^{15}$

Wyatt stated that patients with small edentulous spans bounded by sound teeth are good candidates for RBFPDs. ${ }^{4}$ Although the young are more likely to have sound teeth, debound rates are higher among people under 30 years of age. ${ }^{9}$

In this clinical report, the lateral occlusion of the patient was canine guided. In a 33-month clinical trial, done by Rashid, $\frac{16}{}$ after disclusion of participants with long discrepancies and heavy occlusions, a high success rate has been recognized for posterior FPDs. Imbery ${ }^{17}$ stated that a prosthesis in a mutually protected occlusal scheme should have a better prognosis than one in a group function.

In this case, the framework in the premolar tooth has been extended maximally on the lingual 
aspect of the tooth to improve resistance form and prevent dislodgment of the restoration. Another goal was to create a defined path of insertion for the framework while minimizing the display of metal. $^{4}$

The use of the defined occlusal rests in this case, either on the porcelain fused to metal crown or on the first premolar, has been advocated to provide support or prevent dislodgement toward the gingival aspect. ${ }^{18}$ An in vitro study of 20 extracted premolars after RBFPD rest preparation noted dentin exposure on all specimens. ${ }^{19}$

Base metal alloy, nickel-chromium-beryllium, is preferred over gold alloy due to its enhanced bond to resin cement. ${ }^{\underline{20}}$ In vitro studies showed that Panavia 21 provided an effective bond strength between feldspathic porcelain and base metal alloy with and without load cycling, while no difference were found between Panavia 21 and Scotchbond Multi-Purpose.$^{20,21}$ Panavia 21 reportedly adheres to sandblasted base metal alloy. .2 $^{2}$

Flowable composite could be applied for filling the small lesion in the first premolar because these light-activated, low-viscosity composites have a low modulus of elasticity and are recommended for small, low, or nonbearing restorations. ${ }^{12}$ Campos $^{13}$ showed that, during occlusal loading in class II restorations, flowable composite did not show statistically significant difference among other tested materials, when comparing their performance.

Upon completion of the RBFPD, the patient was satisfied and reported no functional or esthetic problems, while follow-up data over a longer period of time might answer some questions in respect to survival of the RBFPD with a PFM crown as an abutment tooth.

\section{Summary}

This clinical report described the indication, selection of a PFM crowned abutment, and clinical procedures involved in the fabrication of an RBFPD that provided a conservative solution for replacement of one posterior tooth.

\section{Clinical Significance}

RBFPDs can be used successfully in both the anterior and the posterior regions of the mouth to replace one or two missing teeth. However, the survival rate of RBFPDs is still considerably less than that of conventional fixed partial dentures. The principal reason for failure is debonding of the framework from the abutment tooth. The selection of nonmobile abutment teeth, preparation design that enhances retention and resistance form, and tooth bonding technique are critical for success.

\section{References}

1. Chow TW, Chung RW, Chu FC, Newsome PR. Tooth preparations designed for posterior resin-bonded fixed partial dentures: a clinical report. J Prosthet Dent. 2002; 88(6):561-4.

2. Foitzik M, Lennon AM, Attin T. Successful use of a single-retainer all-ceramic resinbonded fixed partial denture for replacement of a maxillary canine: a clinical report. Quintessence Int. 2007; 38(3):241-6.

3. Turker SB, Guvenli SY, Arikan A. Replacement of two mandibular central incisors using a zirconium resin-bonded fixed partial denture: a clinical report. J Prosthet Dent. 2005; 94(6):499-503.

4. Wyatt CC. Resin-bonded fixed partial dentures: what's new? J Can Dent Assoc. 2007; 73(10):933-8.

5. Behr M, Rosentritt M, Handel G. Fiberreinforced composite crowns and FPDs: a clinical report. Int J Prosthodont. 2003; 16(3):239-43.

6. Briggs P, Dunne S, Bishop K. The single unit, single retainer, cantilever resin-bonded bridge. Br Dent J. 1996; 181(10):373-9.

7. Crothers AJ, Wassell RW, Jepson N, Thomason JM. The use of cantilever bridges. Dent Update. 1995; 22(5):190-8.

8. De Kanter RJ, Creugers NH, Verzijden CW, Van't Hof MA. A five-year multi-practice clinical study on posterior resin-bonded bridges. J Dent Res. 1998; 77(4):609-14.

9. Goodacre CJ, Bernal G, Rungcharassaeng $\mathrm{K}$, Kan JY. Clinical complications in fixed prosthodontics. J Prosthet Dent. 2003; 90(1):31-41. 
10. Hussey DL, Linden GJ. The clinical performance of cantilevered resin-bonded bridgework. J Dent. 1996; 24(4):251-6.

11. Roberson TM, Heymann HO, Swift EJ. Sturdevant's art and science of operative dentistry. 5th ed. St. Louis: Mosby; 2006. p. 588.

12. Powers JM, Sakaguchi RL. Craig's restorative dental materials. 12th ed. St. Louis: Mosby; 2006. p. 204.

13. Campos PE, Barceleiro Mde O, SampaioFilho HR, Martins LR. Evaluation of the cervical integrity during occlusal loading of Class II restorations. Oper Dent. 2008; 33(1):59-64.

14. Aboush YE, Estetah N. A prospective clinical study of a multipurpose adhesive used for the cementation of resin-bonded bridges. Oper Dent. 2001; 26(6):540-5.

15. Creugers $\mathrm{NH}$, Käyser $A F$, van 't Hof MA. A meta-analysis of durability data on conventional fixed bridges. Community Dent Oral Epidemiol. 1994; 22(6):448-52.

16. Rashid S, Abidi YA, Hosein T. Success rate of resin bonded restorative dentistry bridges. J Coll Physicians Surg Pak. 2003; 13(12):684-7.

17. Imbery TA, Eshelman EG. Resin-bonded fixed partial dentures: a review of three decades of progress. J Am Dent Assoc. 1996;127(12):1751-60.

18. Emara RZ, Byrne D, Hussey DL, Claffey N. Effect of groove placement on the retention/ resistance of resin-bonded retainers for maxillary and mandibular second molars. J Prosthet Dent. 2001; 85(5):472-8.

19. Bassi GS, Youngson CC. An in vitro study of dentin exposure during resin-bonded fixed partial denture preparation. Quintessence Int. 2004; 35(7):541-8.

20. Walker MP, Spencer P, Eick JD. Effect of simulated resin-bonded fixed partial denture clinical conditions on resin cement mechanical properties. J Oral Rehabil. 2003; 30(8):837-46.

21. Goharian R, Maleknejad F, Salari T, Ghavamnasiri M, Derhami M. Effect of adhesives on bond strength of porcelain veneer to base metal alloy. Quintessence Int. 2002; 33(8):595-9.
22. Czerw RJ, Wakefield CW, Robbins JW, Fulkerson MS. Shear bond strength of composite resin to microetched metal with five newer-generation bonding agents. Oper Dent. 1995; 20(2):58-62.

\section{About the Authors}

Marjaneh Ghavamnasiri DDS, MS (Corresponding Author)

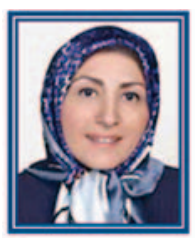

Dr. Ghavamnasiri is a professor and the director of the postgraduate program in the Department of Restorative Dentistry of the Mashhad Dental School and Dental Research Center at Mashhad University of Medical Sciences in Mashhad, Iran.

e-mail: Ghavamnasirim@mums.ac.ir

Fathemeh Maleknejad, DDS, MS

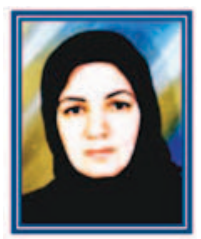

Dr. Maleknejad is an associate professor in the Department of Operative Dentistry, School of Dentistry, Dental Research Center at Mashhad University of Medical Sciences in Mashhad, Iran. Her research interests include tooth bleaching, microleakage, and tooth-colored restorative materials. Dr. Maleknejad is a member of the Iranian Academy of Cosmetic Restorative Dentistry.

e-mail: maleknejadf@mums.ac.ir

Maryam Modabber, DDS

Dr. Modabber is a postgraduate student in the Department of Operative Dentistry at Mashhad Dental School, Mashhad University of Medical Sciences in Mashhad, Iran.

\section{Acknowledgement}

This study was supported by a grant from the Research Council of Mashhad University of Medical Sciences, Iran. 